\title{
Quantum and Thermal Conversion of Solar Energy to Useful Work
}

D. H. Johnson

December 1983

Presented at the Solar Thermal Workshop Atlanta, Georgia

7-8 September 1983

Prepared under Task No. 1387.25

FTP No. 417

Solar Energy Research Institute

A Division of Midwest Research Institute

1617 Cole Bouievard

Golden, Colorado 80401

Prepared for the

U.S. Department of Energy

Contract No. DE-AC02-83CH10093 


\section{NOTICE}

This report was prepared as an account of work sponsored by the United States Government. Neither the United States nor the United States Department of Energy, nor any of their employees, nor any of their contractors, subcontractors, or their employees, makes any warranty, express or implied, or assumes any legal liability or responsibility for the accuracy, completeness or usefulness of any information, apparatus, product or process disclosed, or represents that its use would not infringe privately owned rights. 
QUANTUM AND THERMAL CONVERSION OF SOLAR ENERGY TO USEFUL WORR

\author{
D. H. Johnson
}

\title{
1.0 INTRODUCTION
}

This paper will summarize the results of a thermodynamic analysis of quantum and thermal processes for converting sunlight into useful work. Quantum and thermal processes acting alone as well as combined quantum-thermal processes will be discussed. Two types of combined processes have been analyzed, the thermally coupled process and the thermally decoupled process. These processes were addressed because there is a hope that a combined quantum-thermal conversion system will prove to be cheaper than either system acting separately. A first step in determining cost is to determine maximum system efficiency. The analysis also indicates the concept with the greatest potential so that further efforts can focus on it. Previous analyses of the thermodynamics of quantum and thermal conversion have been performed by Haught (Ref. 1), Bolton (Ref. 2), Ross (Ref.3), and others. This paper will review Haught's analysis and present the results of an extension of this analysis to a thermally decoupled combined quantum/thermal system performed at SERI. Only systems using unconcentrated solar flux will be considered in the present analysis.

\subsection{THERMAC CONVERSION}

Figure 1 is a schematic of a thermal conversion system. Incident radiation is absorbed in a receiver operating at temperature $\mathrm{T}_{\mathrm{T}}$. The receiver is assumed to be perfectly insulated against convective and conductive losses. Some of the absorbed radiation is reradiated to the environment. The rest is converted to heat at temperature $\mathrm{T}_{\mathrm{T}}$. A heat engine converts some of this heat to useful work and rejects the rest to a heat reservoir at the ambient temperature. 
We will assume here and in the rest of this report that the incident radiation is composed of radiation from the sun and from the ambient environment. We will also assume that all sources of radiant energy are black bodies characterized by their temperature. Thus, the intensity of photons from the sun $I_{S}(\nu)$ as a function of frequency $\nu$ is

$$
I_{S}(v)=\frac{2 \pi n^{2} v^{2}}{c^{2}}\left[\exp \left(-h v / k T_{S}\right)-1\right]^{-1} \cdot\left(\frac{R_{S}}{R_{E O}}\right)^{2}
$$

where $n$ is the index of refraction ( $I$ assume $n=1$ ), $C$ is the speed of light $\left(3.00 \times 10^{8} \mathrm{~ms}^{-1}\right), \mathrm{h}$ is Planck's constant $\left(6.63 \times 10^{-30} \mathrm{Js}\right), k$ is Boltzman's constant $\left(1.38 \times 10^{23} \mathrm{JK}^{-1}\right), \mathrm{R}_{\mathrm{S}}$ is the radius of the sun $\left(6.95 \times 10^{8} \mathrm{~m}\right), \mathrm{R}_{\mathrm{EO}}$ is the radius of the earth's orbit $\left(1.49 \times 10^{11} \mathrm{~m}\right)$, and $T_{S}$ is the temperature of the sun $(6000 \mathrm{~K})$. The factor $\left(R_{S} / R_{E O}\right)^{2}$ is the solid angle subtended by the sun and represents the fraction of the radiant energy emitted by the sun which is incident on the receiver. Similarly, the intensity of photons from the ambient environment $I_{A}(v)$ is

$$
I_{A}(\nu)=\frac{2 \pi n^{2} \nu^{2}}{c^{2}}\left[\exp \left(-h v / k T_{A}\right)-1\right]^{-1}\left[1-\left(\frac{R_{s}}{R_{E O}}\right)^{2}\right]
$$

where $T_{A}$ is the ambient temperature $(300 \mathrm{~K})$ and the factor $\left[1-\left(R_{S} / R_{E 0}\right)^{2}\right]$ accounts for the blocking of the environment by the sun. The intensity of light reradiated from the receiver is

$$
I\left(\nu, T_{T}\right)=\frac{2 \pi n^{2} \nu^{2}}{c^{2}}\left[\exp \left(-h \nu / k T_{T}\right)-1\right]^{-1}
$$

We will assume that the heat engine absorbs heat from a reservoir at temperature $\mathrm{T}_{\mathrm{T}}$ (the receiver), rejects heat to a reservoir at temperature $\mathrm{T}_{\mathrm{A}}$ (the ambient environment), and converts the difference into work with a Carnot efficiency given by $\left(1-\mathrm{T}_{\mathrm{A}} / \mathrm{T}_{\mathrm{T}}\right)$. 
The efficiency of the thermal conversion process is then given by

$$
\eta_{T}=\frac{\int_{0}^{\infty}\left[I_{S}(\nu)+I_{A}(\nu)-I\left(\nu, T_{T}\right)\right] h \nu\left(1-\frac{T_{A}}{T_{T}}\right) \sigma\left(\nu, T_{T}\right) d \nu}{\int_{0}^{\infty} I_{S}(\nu) h v d \nu}
$$

where the factor in brackets represents the number of photons absorbed per unit area of the receiver, $h \nu$ is the energy per photon of frequency $\nu$, $\left(1-T_{A} / T_{T}\right.$ ) is the Carnot efficiency of conversion of thermal energy to work, and $\sigma\left(\nu, \mathrm{T}_{\mathrm{T}}\right)$ is the absorptivity of the receiver.

According to the principle of detailed balancing the absorptivity equals the emissivity. Since the spectrum of incident photons from the sun is peaked at much higher frequency than the spectrum of the photons emitted from the receiver, conversion efficiency will be improved by choosing an absorptivity that is large at high frequencies and small at low frequencies. We will consider a step function absorptivity such that

$$
\sigma\left(\nu, \mathrm{T}_{\mathrm{T}}\right)=\begin{aligned}
& 0 \nu<\nu_{\mathrm{T}}\left(\mathrm{T}_{\mathrm{T}}\right) \\
& 1 \nu>\nu_{\mathrm{T}}\left(\mathrm{T}_{\mathrm{T}}\right)
\end{aligned}
$$

The conversion efficiency is a function of $T_{T}$ and $\nu_{T}$. The temperature that produces the maximum conversion efficiency for each $\nu_{T}$ is plotted as a function of $\nu_{T}$ in Figure 2. The conversion efficiency produced by using the optimum temperature for each $\nu_{T}$ is also plotted as a function of $\nu_{T}$ in Figure 2. The maximum possible conversion efficiency is $54 \%$ and occurs at a temperature of $862 \mathrm{~K}$ and an absorption cutoff of $2.22 \times 10^{14} \mathrm{~s}^{-1}$ (a wavelength $\lambda$ of $\left.1.35 \times 10^{-16} \mathrm{~m}\right)$. Notice that the optimum temperature is essentially proportional to the absorption cutoff frequency except near $v_{\mathrm{T}}=0$ where $\mathrm{T}_{\mathrm{T}} \approx 400 \mathrm{~K}$. 


\subsection{QUANTUM CONVERSION}

Figure 3 is a schematic of a quantum conversion system. The entire system is perfectly insulated against conductive and convective heat loss and operates at temperature $\mathrm{T}_{\mathrm{Q}}$. The receiver absorbs incident radiation, some of which is reradiated to the environment. However, the absorption process is different than in the thermal case. Instead of being completely converted to heat, a portion $\mu$ of the energy of each absorbed photon is converted to the available energy of an excited state with the remaining energy dissipated as heat at the temperature $\mathrm{T}_{\mathrm{Q}}$. The population of the excited state is not in equilibrium with the receiver at temperature $\mathrm{T}_{\mathrm{Q}}$. It can be thought of as the population that would exist in equilibrium with its surroundings at a higher temperature. The population of the excited state is separated from the surrounding unexcited material (and possibly stored for a time). A quantum engine then converts the population of the excited state to unexcited material, recovering the available energy $\mu$ as useful work in the process. Notice that the separation step is unavoidable. If this step were not performed independently, the process of converting the population of the excited state to unexcited material in the presence of material that was never excited would effectively result in separating the population of the excited state from that material.

The intensity of photons incident from the sun and the ambient environment is as discussed in the preceding section. The requirement that the quantum receiver, which converts $(h \nu-\mu)$ of the energy of each absorbed photon to heat at temperature $T_{Q}$, be in equilibrium with the radiation that it emits, leads to the following intensity of light emitted from the receiver:

$$
I\left(\nu, \mu, T_{Q}\right)=\frac{2 \pi n^{2} \nu^{2}}{c^{2}}\left\{\exp \left[-(h \nu-\mu) / k T_{Q}\right]-1\right\}^{-1} \text {. }
$$

We will assume that the quantum engine converts all of the available energy of the excited state into useful work.

The efficiency of the quantum conversion process is then given by 


$$
\eta_{Q}=\frac{\int_{0}^{\infty}\left[I_{S}(\nu)+I_{A}(\nu)-I\left(\nu, \mu, T_{Q}\right)\right] \mu \sigma\left(\nu, \mu, T_{Q}\right) d \nu}{\int_{0}^{\infty} I_{S}(\nu) h v d \nu}
$$

where, as before, the factor in brackets represents the number of photons absorbed per unit area of the receiver, $\mu$ is the portion of the energy of each absorbed photon converted to available energy of the excited state, and $\sigma\left(\nu, \mu, \mathrm{T}_{\mathrm{Q}}\right)$ is the absorptivity of the receiver. The absorptivity of the quantum receiver is determined by the quantum process. We will assume a single photon excitation process characterized by a threshold frequency below which absorption and excitation of the medium does not occur. In this case,

$$
\sigma\left(\nu, \mu, \mathrm{T}_{\mathrm{Q}}\right)=\begin{aligned}
& 0 \nu<\nu_{\mathrm{Q}}\left(\mu, \mathrm{T}_{\mathrm{Q}}\right) \\
& 1 \nu>\nu_{\mathrm{Q}}\left(\mu, \mathrm{T}_{\mathrm{Q}}\right)
\end{aligned}
$$

Notice that to have a finite emission spectrum, the threshold for emission must always be less than the threshold for absorption $h \nu_{Q}$. The difference between $h \nu_{Q}$ and $\mu$ is the heat of mixing, released during separation of the population of the excited state from the unexcited states. The fact that $\mu$ is less than $h v_{Q}$ is required by the second law of thermodynamics and in that sense, $\mu$ plays the same role as the Carnot efficiency of a heat engine.

The conversion efficiency is a function of $\mu, T_{Q}$, and $\nu_{Q}$. By inspection of Eq. 7 , it is obvious that the maximum quantum conversion efficiency occurs at the lowest possible temperature of the receiver, which is the ambient temperature $\mathrm{T}_{\mathrm{A}}$. Henceforth, we will assume that for a pure quantum conversion system $\mathrm{T}_{\mathrm{Q}}=\mathrm{T}_{\mathrm{A}}$. If we find the available energy that maximizes the conversion efficiency for a given threshold frequency $\nu_{Q}$ and then do this again for a range of $\nu_{Q}$ we get the results shown in Figure 4. The maximum possible conversion efficiency is $31 \%$ and occurs at an available energy of $1.5 \times 10^{-12}$ erg and a threshold frequency of $3.16 \times 10^{14} \mathrm{~s}^{-1}\left(\lambda=9.5 \times 10^{-7} \mathrm{~m}\right)$. Also, notice that the optimum $\mu$ is approximately proportional to $\nu_{Q}$ and that at $\nu_{Q}=0$, the optimum $\mu$ is zero. 


\subsection{THERMALLY COUPLED QUANTUM-THERMAL CONVERSION}

Figure 5 is a schematic of a thermally coupled combined quantum-thermal conversion system. A receiver operating at a temperature $\mathrm{T}_{Q}$ absorbs incident radiation and converts a portion $\mu$ of the energy of each absorbed photon to the available energy of an excited state. The remaining energy $(h \nu-\mu)$ is rejected as waste heat at the temperature of the quantum converter $T_{Q}$. This waste heat is used as the input to a bottoming thermal cycle.

The incident radiation spectrum is the same as previously discussed and the emitted radiation spectrum is the same as for a quantum system. The quantum engine converts all of the available energy $\mu$ into useful work while the heat engine converts the remaining energy $(h \nu-\mu)$ of each photon into useful work with the Carnot efficiency $\left(1-\mathrm{T}_{\mathrm{A}} / \mathrm{T}_{\mathrm{Q}}\right)$.

The efficiency of the thermally coupled combined quantum-thermal conversion process is given by

$$
\eta_{Q-T}=\frac{\int_{0}^{\infty}\left[I_{S}(\nu)+I_{A}(\nu)-I\left(\nu, \mu, T_{Q}\right)\right]\left[\mu+(h \nu-\mu)\left(1-\frac{T_{A}}{T_{Q}}\right)\right] \sigma\left(\nu, \mu, T_{Q}\right) d \nu}{\int_{0}^{\infty} I_{S}(\nu) h v d \nu}
$$

where all the symbols have been previously defined and $\sigma\left(\nu, \mu, T_{Q}\right)$ is, as before, the absorptivity of the receiver. We will assume, as previously, that

$$
\sigma\left(\nu, \mu, \mathrm{T}_{\mathrm{Q}}\right)=\begin{aligned}
& 0 \nu<\nu_{\mathrm{Q}}\left(\mu, \mathrm{T}_{\mathrm{Q}}\right) \\
& 1 \nu>\nu_{\mathrm{Q}}\left(\mu, \mathrm{T}_{\mathrm{Q}}\right)
\end{aligned},
$$

where $\nu_{Q}$ is, again, the threshold frequency below which absorption and excitation of the quantum process does not occur.

The conversion efficiency is a function of $\mu, T_{Q}$, and $\nu_{Q}$. Because of the appearance of $\mathrm{T}_{\mathrm{Q}}$ in the Carnot efficiency factor as well as the emitted radiation spectrum, the maximum efficiency will not in general occur at $T_{Q}=T_{A}$. 
If we find the available energy that maximizes the efficiency for a given $\nu_{0}$ and $T_{Q}$ and then plot contours of the efficiency for this value of $\mu$ versus $v_{Q}$ and $\mathrm{T}_{Q}$, we get the results shown in Figure 6 . The curve that runs from the lower left of the figure to the upper right represents the conditions for which $\mu=0$. Along this curve, the quantum part of the system produces no useful work and the combined system is equivalent to a pure thermal system. Efficiency and corresponding temperature plotted versus threshold frequency along this curve would reproduce Figure 2. Below this curve $\mu$ would be less than zero, implying that the system was supplying energy to the incident radiation. Along the vertical axis at $T_{Q}=300 \mathrm{~K}=\mathrm{T}_{\mathrm{A}}$, the Carnot efficiency of the thermal conversion part of the system is zero and the combined system is equivalent to a pure quantum system. Efficiency and available energy plotted versus threshold frequency along the vertical axis would reproduce Figure 4. The region between the vertical axis and the curve $\mu=0$ represents combinations of $\nu_{Q}$ and $T_{Q}$ for which the system has both a quantum and a thermal part. It is instructive to explore further optimization of the system along two paths in this region. If we find the temperature that maximizes the efficiency for a given $\nu_{Q}$, then we will trace out the lower portion of the $\mu=0$ curve. This approach to optimization leads to a pure thermal system. If we find the threshold frequency that maximizes the efficiency for a given $\mathrm{T}_{Q}$, then we trace out the dashed curve in Figure 6. This approach to optimization leads to systems with both a quantum and a thermal conversion part.

If we plot overall system efficiency $\eta_{Q-T}$ and the individual contributions to overall efficiency of the quantum part $\eta_{Q}$ and the thermal part $\eta_{T}$ versus $T_{Q}$ along this dashed curve, we get the results presented in Figure 7. The overall system efficiency increases from that of a pure quantum system at $\mathrm{T}_{\mathrm{Q}}=300 \mathrm{~K}\left(\eta_{\mathrm{Q}-\mathrm{T}}=\eta_{\mathrm{T}}=0.31\right)$ to that of a pure thermal system at $\mathrm{T}_{\mathrm{Q}}=862 \mathrm{~K}\left(\eta_{\mathrm{Q}-\mathrm{T}}=\eta_{\mathrm{T}}=0.54\right)$. The contribution of the quantum part of the system to overall efficiency decreases monotonically from 0.31 at $\mathrm{T}_{\mathrm{Q}}=300 \mathrm{~K}$ to zero at $\mathrm{T}_{\mathrm{Q}}=862 \mathrm{~K}$ while that of the thermal part increases from zero at $300 \mathrm{~K}$ to 0.54 at $862 \mathrm{~K}$. The quantum and thermal parts make equal contribution to overall efficiency at $\mathrm{T}_{\mathrm{Q}}=450 \mathrm{~K}$ where each is $21 \%$. 


\subsection{THERMALLY DECOUPLED QUANTUM/THERMAL CONVERSION}

Figure 8 is a schematic of a thermally decoupled combined quantum/thermal conversion system. The spectrum of incident radiation is split into a low frequency part and a high frequency part by a dichroic beam splitter (alternatively, the quantum process, which absorbs high frequencies but passes low frequencies, could be placed in front of the thermal process). The high frequency part of the incident spectrum is directed to a quantum receiver operating at temperature $\mathrm{T}_{\mathrm{Q}}$ while the low frequency part is directed to a thermal receiver operating at temperature $\mathrm{T}_{\mathrm{T}}$. The quantum and thermal parts of the system then each operate upon their part of the spectrum as previously described.

The efficiency of the thermally decoupled combined quantum/thermal conversion process is given by

$$
\begin{aligned}
\eta_{Q / T}= & \left\{\int_{0}^{\infty}\left[I_{S}(\nu)+I_{A}(\nu)-I_{Q}\left(\nu, \mu, T_{Q}\right)\right] \mu \sigma_{D B S}\left(\nu, \mu, T_{Q}, T_{T}\right) d \nu\right. \\
& +\int_{0}^{\infty}\left[I_{S}(\nu)+I_{A}(\nu)-I_{T}\left(\nu, T_{T}\right)\right] h \nu\left(1-\frac{T_{A}}{T_{T}}\right) \\
& {\left.\left[1-\sigma_{D B S}\left(\nu, \mu, T_{Q}, T_{T}\right)\right] \sigma_{T}\left(\nu, T_{T}\right) d \nu\right\} \div \int_{0}^{\infty} I_{S}(\nu) h \nu d \nu }
\end{aligned}
$$

where $\sigma_{\mathrm{DBS}}\left(\nu, \mu, \mathrm{T}_{\mathrm{Q}}, \mathrm{T}_{\mathrm{T}}\right)$ is the transmissivity of the dichroic beam splitter that is chosen to match the absorptivity of the quantum process. As before, we will assume that

$$
\sigma_{\text {DBS }}\left(\nu, \mu, \mathrm{T}_{\mathrm{Q}}, \mathrm{T}_{\mathrm{T}}\right)=\begin{aligned}
& 0 \nu<v_{\mathrm{Q}}\left(\mu, \mathrm{T}_{\mathrm{Q}}, \mathrm{T}_{\mathrm{T}}\right) \\
& 1 \nu>\nu_{\mathrm{Q}}\left(\mu, \mathrm{T}_{\mathrm{Q}}, \mathrm{T}_{\mathrm{T}}\right)
\end{aligned}
$$

where $v_{Q}$ is the excitation threshold of the quantum system. The absorptivity of the thermal receiver is given by $\sigma_{\mathrm{T}}$. For the reasons discussed in Section 2.0 , it is desirable to not absorb very low frequencies in the thermal receiver. We will assume that 


$$
\sigma_{\mathrm{T}}\left(\nu, \mathrm{T}_{\mathrm{T}}\right)=\begin{aligned}
& 0 \nu>\nu_{\mathrm{T}}\left(\mathrm{T}_{\mathrm{T}}\right) \\
& 1 \nu<\nu_{\mathrm{T}}\left(\mathrm{T}_{\mathrm{T}}\right)
\end{aligned}
$$

In Eq. 11, $I_{Q}$ is the spectrum of light emitted from the quantum receiver as given by Eq. 6 and $I_{T}$ is that emitted from the thermal receiver as given by Eq. 3. All other terms have been defined previously.

The efficiency of the thermally decoupled combined quantum/thermal conversion system is a function of $\mu, T_{Q}, T_{T}, \nu_{Q}$, and $\nu_{T}$. Inspection of $\mathrm{Eq}$. 11 shows that the maximum system efficiency occurs at the lowest possible temperature of the quantum receiver which is the ambient temperature. We will assume in the following that $T_{Q}=T_{A}$. If we now examine maximizing the overall conversion efficiency with respect to the remaining parameters, we find that the conditions on $\mu$ that result in maximizing $\eta_{Q / T}$ for a given $\nu_{Q}, T_{T}$, and $\nu_{T}$ are the same as for a pure quantum system (the optimum $\mu$ is a function only of $\nu_{Q}$ and is that given in Figure 4). Similarly, the conditions on $\nu_{T}$ that result in maximizing $\eta_{Q / T}$ for a given $\nu_{Q}, T_{T}$, and $\mu$ are the same as for a pure thermal system (the optimum $\nu_{T}$ is a function only of $T_{T}$ and is that given in Figure 2).

If we now use these optimum values of $\mu$ as a function of $\nu_{Q}$ and $\nu_{T}$ as a function of $\mathrm{T}_{\mathrm{T}}$, we get the results shown in Figure 9. The curve that runs from the lower left portion of the figure to the upper right represents the case where $\nu_{T}=\nu_{Q}$ and $\mu=0$. Along this curve, both the thermal and the quantum parts of the system have zero conversion efficiency. Below this curve $\nu_{T}$ would be greater than $\nu_{Q}$ and the thermal part of the system would not work. Also, below this curve $\mu$ would be less than zero and the quantum part of the system would be supplying energy to the incident radiation. Along the vertical axis at $\mathrm{T}_{\mathrm{T}}=300, \mathrm{~K}=\mathrm{T}_{\mathrm{A}}$, the Carnot conversion efficiency of the thermal conversion part of the system is zero and the combined system is equivalent to a pure quantum system. Efficiency and available energy plotted versus threshold frequency along the vertical axis would reproduce Figure 4. The region between the vertical axis and the $\nu_{Q}=\nu_{T}, \mu=0$ curve represents combinations of $\nu_{Q}$ and $T_{T}$ for which the system has both a quantum and a thermal part. It is instructive to explore further optimization of the system along two paths 
in this region. If we find the temperature that maximizes the efficiency for a given $v_{Q}$, then we will trace out the dotted line in Figure 9. If we find the threshold frequency that maximizes the efficiency for a given $\mathrm{T}_{\mathrm{T}}$, then we will trace out the dashed curve in Figure 9. Along both these curves, the system has both a quantum and a thermal part.

Consider the behavior of the system along the curve of optimum temperature (the dotted curve of Figure 9) as shown in Figure 10. The overall system efficiency increases along this curve from zero at $T_{T}=300 \mathrm{~K}, \nu_{Q}=0 \mathrm{~s}^{-1}$ to $54.7 \%$ at $\mathrm{T}_{\mathrm{T}}=834 \mathrm{~K}, \nu_{\mathrm{Q}}=7.83 \times 10^{14} \mathrm{~s}^{-1}\left(\lambda=3.8 \times 10^{-7} \mathrm{~m}\right)$. This is a higher conversion efficiency than that achieved by either a quantum or a thermal system alone, or by a thermally coupled combined quantum-thermal system. The contribution of the thermal part of the system increases along this curve from zero at $\mathrm{T}_{\mathrm{T}}=300 \mathrm{~K}$ to $46.3 \%$ at $\mathrm{T}_{\mathrm{T}}=834 \mathrm{~K}$, the temperature of each system efficiency. The contribution of the quantum part of the system first increases from zero at $T_{T}=300 \mathrm{~K}, \nu_{Q}=0 \mathrm{~s}^{-1}$ to a peak of $31 \%$ at $\mathrm{T}_{\mathrm{T}}=625 \mathrm{~K}$, $\nu_{Q}=3.16 \times 10^{14} \mathrm{~s}^{-1}\left(\lambda=9.52 \times 10^{-7} \mathrm{~m}\right)$ and then decreases, but still contributes $8.4 \%$ at $\mathrm{T}_{\mathrm{T}}=834 \mathrm{~K}$, the temperature of peak system efficiency. The quantum and thermal parts make equal contributions at $\mathrm{T}_{\mathrm{T}}=700 \mathrm{~K}$ where each is $26 \%$.

Now consider the behavior of the system along the curve of optimum threshold frequency (the dashed curve in Figure 9) as shown in Figure 11. This curve is analogous to the dashed curve in Figure 7 for a thermally coupled system. The overall system efficiency increases along this curve from that of a pure quantum system at $\mathrm{T}_{\mathrm{T}}=300 \mathrm{~K}\left(\eta_{\mathrm{Q} / \mathrm{T}}=31 \%\right)$ to a peak of $54.7 \%$ at $\mathrm{T}_{\mathrm{T}}=834 \mathrm{~K}$ and $\nu_{Q}=7.83 \times 10^{14} \mathrm{~s}^{-1}\left(\lambda=3.8 \times 10^{-7} \mathrm{~m}\right)$. This is, of course, the same maximum system efficiency reached along the path of optimum $\mathrm{T}_{\mathrm{T}}$ discussed in the preceding paragraph. The contribution of the thermal part of the system along this curve first increases from zero at $\mathrm{T}_{\mathrm{T}}=300 \mathrm{~K}$ until it reaches $46.3 \%$ at $\mathrm{T}_{\mathrm{T}}=834 \mathrm{~K}$, the temperature of peak system efficiency, and continues to increase until it reaches $51.5 \%$ at $\mathrm{T}_{\mathrm{T}}=858 \mathrm{~K}, \nu_{\mathrm{Q}}=10^{16} \mathrm{~s}^{-1}\left(\lambda=3 \times 10^{-8} \mathrm{~m}\right)$. After this, it decreases monotonically to zero at high temperature. The contribution of the quantum part decreases monotonically from an efficiency of $31 \%$ at $\mathrm{T}_{\mathrm{T}}=300 \mathrm{~K}$ to zero at high temperature. However, as discussed before, 
the quantum part contributes $8.3 \%$ at the conditions of maximum system efficiency.

\subsection{CONCLUSIONS AND RECOMMENDATIONS}

For a pure quantum system compared to a pure thermal system, optimum thermal conversion is more efficient (by a factor of 1.74) than optimum quantum conversion. This is largely because only a fixed portion of each photon absorbed by the quantum process is available to do work. The rest is immediately thermalized and rejected as waste heat. In a thermal process, the full energy of each photon is available for conversion to useful work with Carnot efficiency. On the other hand, optimum thermal conversion occurs at high temperature $\left(\mathrm{T}_{\mathrm{T}}=862 \mathrm{~K}\right)$. Since costs generally increase with temperature, it may be that a quantum conversion system will be cheaper than a thermal conversion system despite its lower efficiency.

For a thermally coupled combined quantum-thermal conversion system, the peak efficiency of $54 \%$ occurs at $T_{Q}=862 \mathrm{~K}, \quad \nu_{Q}=2.22 \times 10^{14} \mathrm{~s}^{-1}$ $\left(\lambda=1.35 \times 10^{-7} \mathrm{~m}\right), \mu=0$. Under these conditions, the contribution of the quantum part of the system is zero. From an efficiency point of view, thermally coupling quantum and thermal conversion processes does not favor quantum conversion. However, since costs generally increase with temperature, the cheapest system may operate at a temperature below that of maximum system efficiency. At temperatures between $300 \mathrm{~K}$ and $862 \mathrm{~K}$, the thermally coupled combined system has a higher efficiency than either a pure quantum system or a pure thermal system operating alone and may be cheaper than either operating alone.

However, the thermally coupled system severely penalizes the quantum component because the thermal component has a higher peak efficiency achieved at high temperature where the quantum component has a very low efficiency. One manifestation of the penalties thermal coupling imposes on the quantum component is demonstrated by the latitude one has in choosing system parameters without greatly affecting overall efficiency. In Figure 6, the parameter that most 
directly affects the quantum component is $\nu_{Q}$, displayed along the vertical axis, while the parameter that most directly affects the thermal component is $\mathrm{T}_{\mathrm{Q}}$, displayed along the horizontal axis. The contours of constant overall system efficiency are generally parallel to the horizontal axis; i.e., $T_{Q}$ can be varied over a considerable range without appreciably affecting overall system efficiency, while a small variation in $\nu_{Q}$ usually has a much stronger effect on overall system efficiency.

For a thermally decoupled combined quantum/thermal conversion system, the peak efficiency is greater ( $54.7 \%$ versus $54 \%$ ) and occurs at a lower temperature ( $834 \mathrm{~K}$ versus $862 \mathrm{~K}$ ) than the thermally coupled system. At peak system efficiency, the thermal part contributes $46.3 \%$ and the quantum part contributes $8.3 \%$, unlike the thermally coupled system for which the quantum component contributes nothing under conditions of peak efficiency. The value of $\nu_{Q}$ under conditions of peak efficiency is $7.83 \times 10^{14} \mathrm{~s}^{-1}\left(\lambda=3.8 \times 10^{-7} \mathrm{~m}\right)$. This is in the near ultraviolet. In other words, thermodynamics requires that this system portions the ultraviolet light to the quantum conversion part and the rest of the solar spectrum to the thermal conversion part. It happens that real quantum conversion processes often have excitation thresholds in the ultraviolet, while ultraviolet light is usually considered harmful to the components of a real thermal system (i.e., ultraviolet light causes devitrification of quartz, a material otherwise well suited for the window of a hightemperature receiver).

The points just discussed demonstrate that the thermally decoupled system is much kinder to the quantum component than the thermally coupled system. This is because the thermally decoupled system allows the quantum component to operate at ambient temperature. This is further illustrated by the latitude one has in choosing system parameters without greatly affecting overall efficiency. In Figure 9, the parameter that most directly affects the quantum component is $\nu_{Q}$, displayed along the vertical axis, while the parameter that most directly affects the thermal component is $\mathrm{T}_{\mathrm{T}}$, displayed along the horizontal axis. The contours of constant system efficiency generally lie parallel to the vertical axis; i.e., $\nu_{Q}$ can be varied over a considerable range without appreciably affecting overall system efficiency, while a small varia- 
tion in $\mathrm{T}_{\mathrm{T}}$ has a much stronger effect on overall system efficiency. Since the temperature of the thermal conversion part of the system can be controlled by the rate of heat transfer while the excitation threshold of the quantum system is not so readily controlled, this aspect of the thermally decoupled system has important practical implications.

Another indication of the greater role the quantum component plays in a thermally decoupled system than in a thermally coupled system is seen by considering the conditions under which the quantum and thermal part contribute equally to the overall system efficiency of each type of system. This occurs for a thermally coupled system at $\mathrm{T}_{\mathrm{Q}}=150 \mathrm{~K}$ where each part contributes $21 \%$ and for a thermally decoupled system (along the path of optimum temperature) at $\mathrm{T}_{\mathrm{T}}=700 \mathrm{~K}$ where each contributes $26 \%$.

It is clear from the above discussion that the thermally decoupled combined quantum/thermal conversion system has considerable potential. However, there is still a large degree of uncertainty about the best operational conditions for the system and about its competitive position relative to the other types of systems. One important operational factor not yet considered is the affect of concentrating the incident sunlight. It is known that concentrating the incident sunlight, with other system parameters fixed, improves the performance of pure quantum and pure thermal conversion systems. As the concentration is increased, heat must be transferred out of the system at an increasing rate to keep temperature constant. In a thermally decoupled system, the quantum part is operating near ambient temperature while the thermal part is operating at high temperature. Thus, it will be harder to transfer heat out of the quantum part than out of the thermal part. This means that the optimum operating conditions may call for a different degree of concentration for the two parts. Other operational factors that determine system configuration also need to be examined. Guided by this analysis a concept for a practical system should be developed. This concept should include all factors of importance to a real system such as the actual quantum process to be used, the limitations of real dichroic beam splitters, the actual thermal process to be used, etc. Finally, the concept should be laid out in enough detail to estimate cost and this cost should be compared to the cost of competitive systems. 


\subsection{REFERENCES}

1. Haught, A. F., 1984, "Physics Considerations of Solar Energy Conversion," to be published in the ASME Trans., Journal of Solar Energy Engineering.

2. Bolton, J. R., 1978, "Solar Fuels," Science 202, No. 4359, 705.

3. Ross, R. T., and T. L. Hsiao, 1979, "Limits on the Yield of Photochemical Solar Energy Conversion," J. App1. Phys., 48, No. 11, 4783.

\subsection{ACKNOWLEDGMENTS}

The author would like to acknowledge the contribution of all who attended the Solar Thermal Fuels and Chemicals Workshop run by SERI in January 1983. Discussions at that workshop provided the original stimulation for the new work reported here. In particular, discussions with $\mathrm{Dr}$. Alan Haught and Prof. Jim Bolton were most helpful. 
Figure 1. Thermal Conversion

Figure 2. Conversion Bfficiency for Thermal System

Figure 3. Quantum Conversion

Figure 4. Conversion Efficiency for Quantum System

Figure 5. Thermally Coupled Combined Quantum-Thermal Conversion

Figure 6. Conversion Bfficiency for Thermally Coupled Combined Quantum-Thermal System

Figure 7. Split Between Quantum and Thermal Conversion for Thermally Coupled Combined System

Figure 8. Thermally Decoupled Combined Quantum/Thermal Conversion

Figure 9. Conversion Efficiency for Thermally Decoupled Combined Quantum/Thermal System

Figure 10. Split Between Quantum and Thermal Conversion for a Thermally Decoupled Combined System

Figure 11. Thermally Decoupled Combined System Efficiency for Optimum Quantum Bxcitation Frequency 


\section{Thermal Conversion}

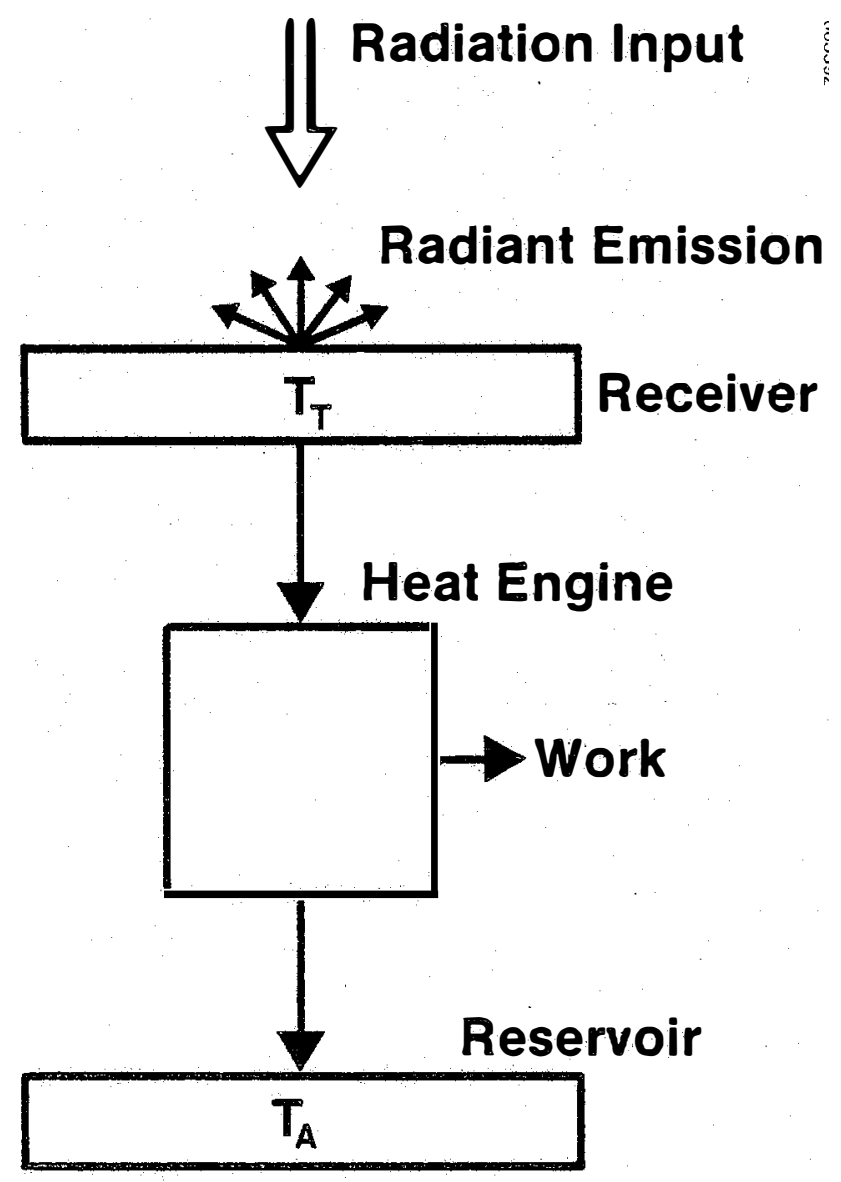

Thermal System

Figure 1. 


\section{Conversion Efficiency for Thermal System}

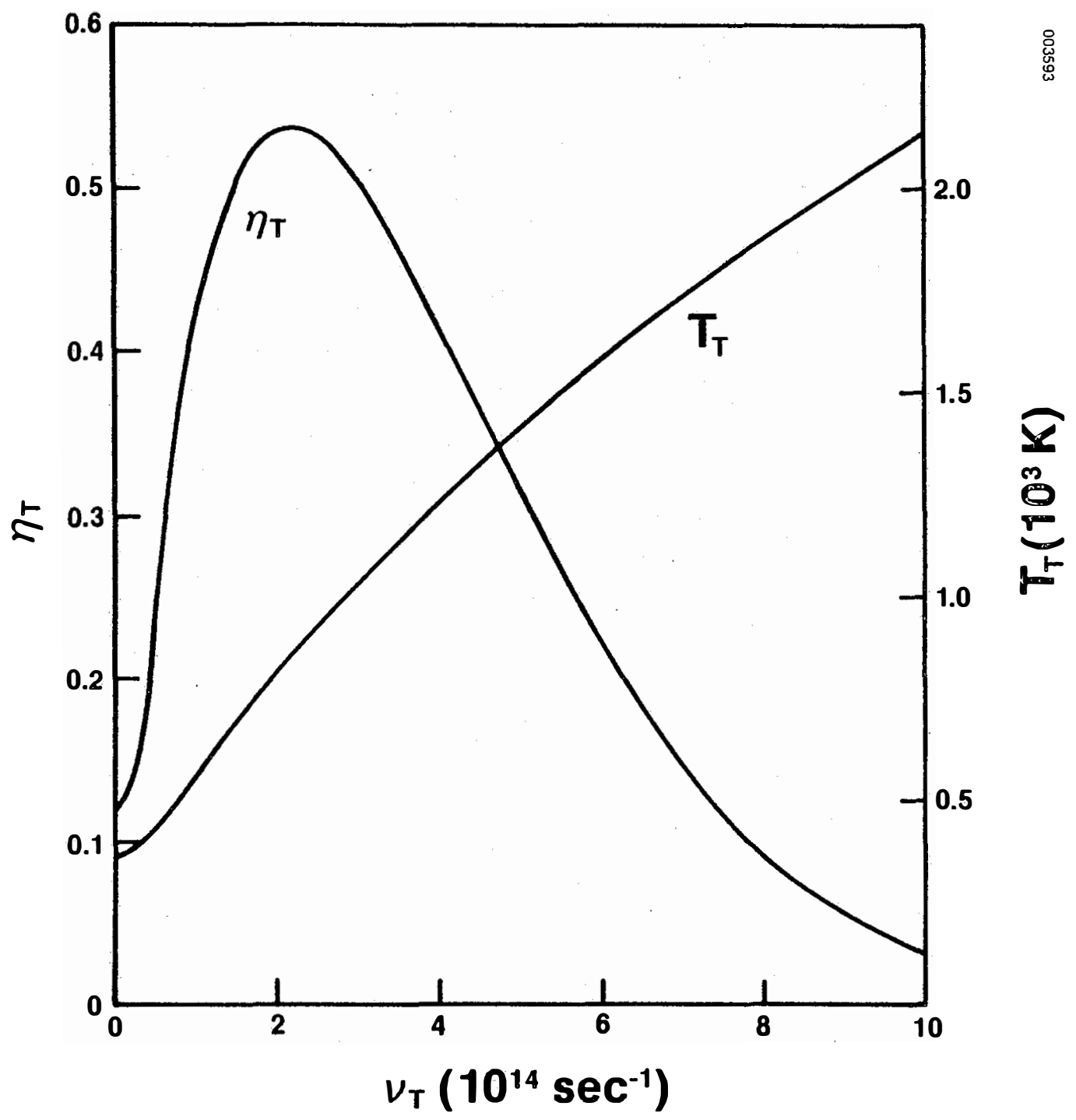

From: Physics Considerations of Solar Energy Conversion by Alan Haught, United Technologies

Figure 2. 


\section{Quantum Conversion}

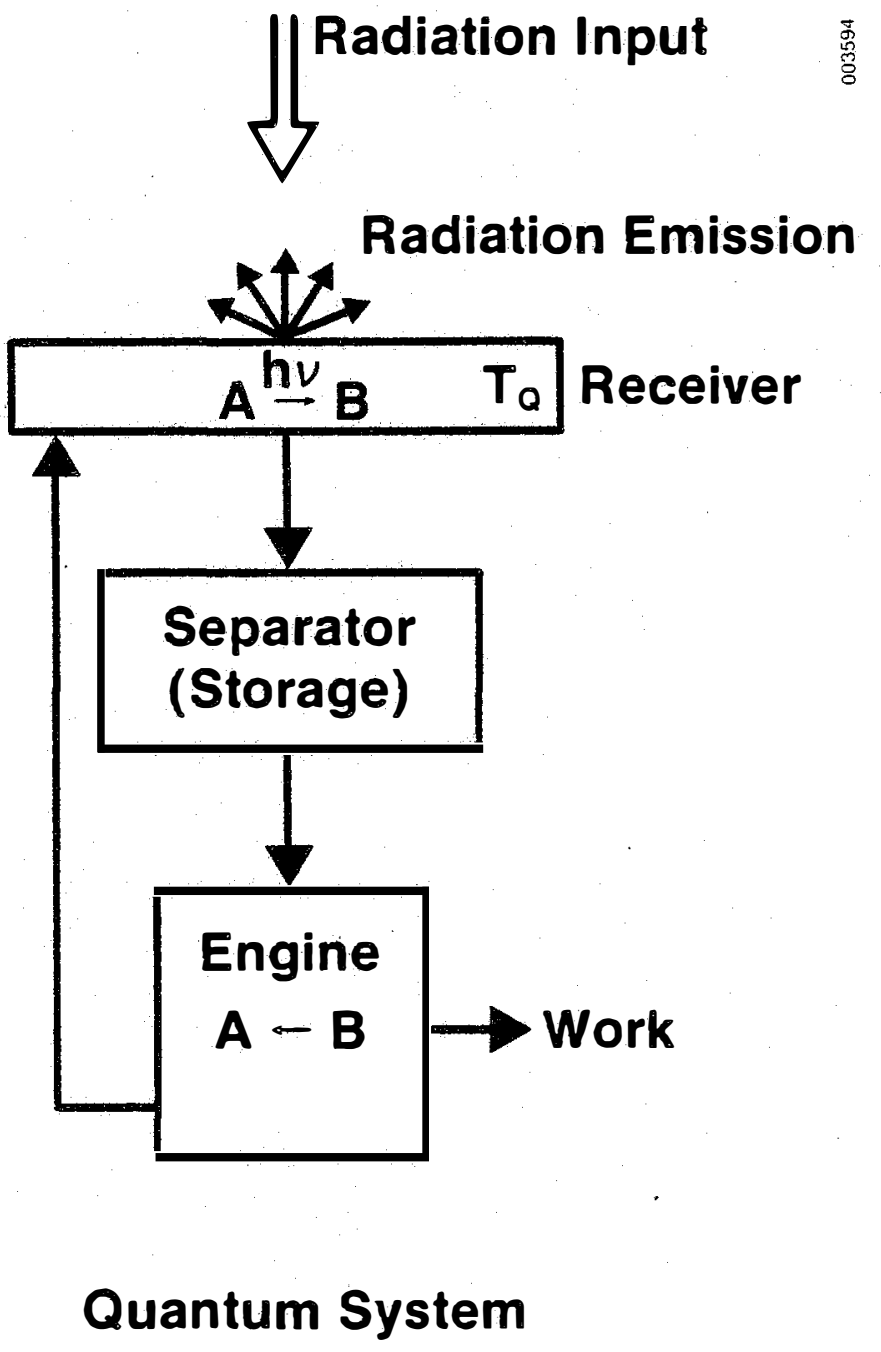

Figure 3. 


\section{Conversion Efficiency for Quantum System}

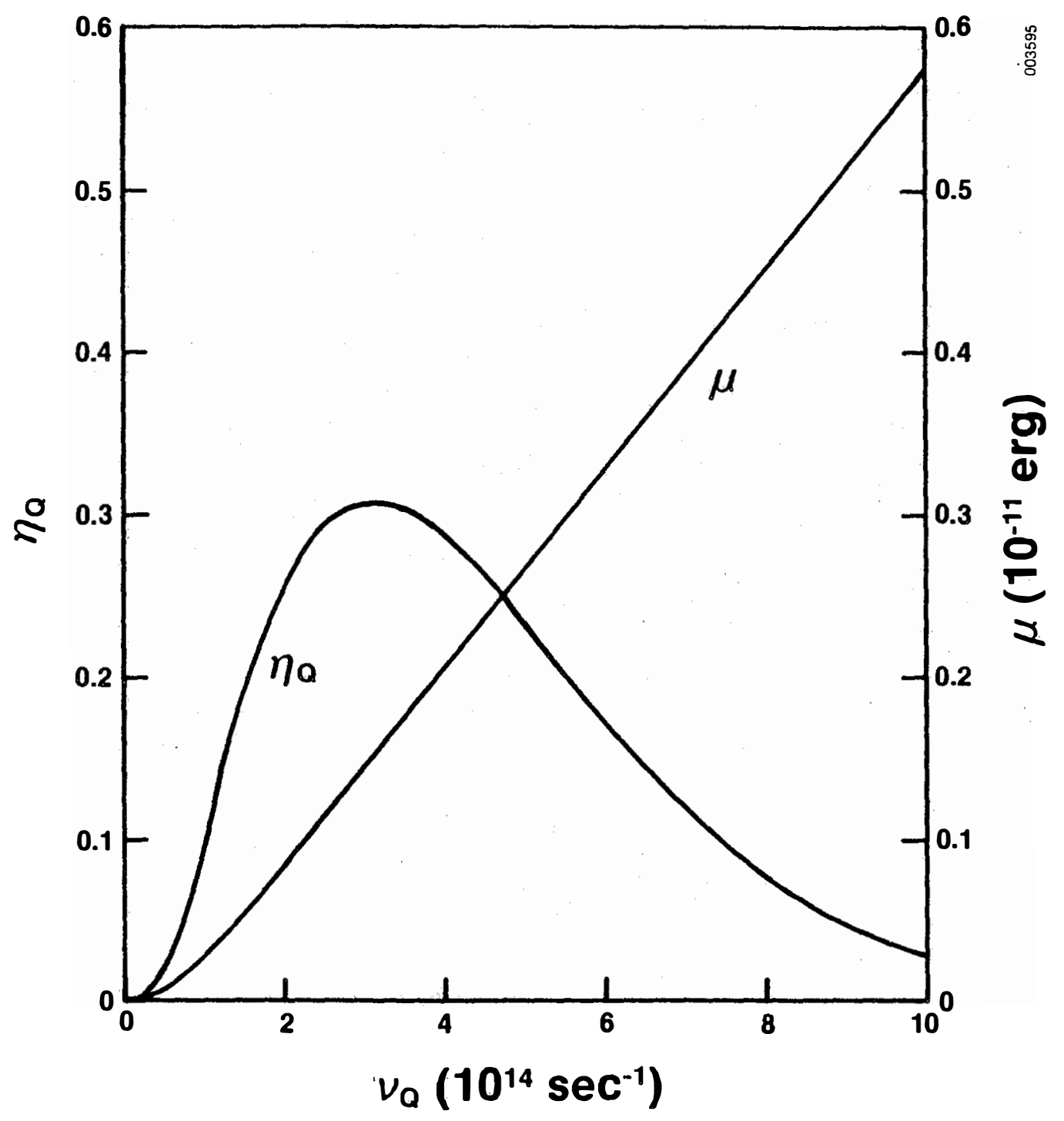

From: Physics Considerations of Solar Energy Conversion by Alan Haught, United Technologies.

Figure 4. 


\section{Thermally Coupled Combined Quantum-Thermal Conversion}

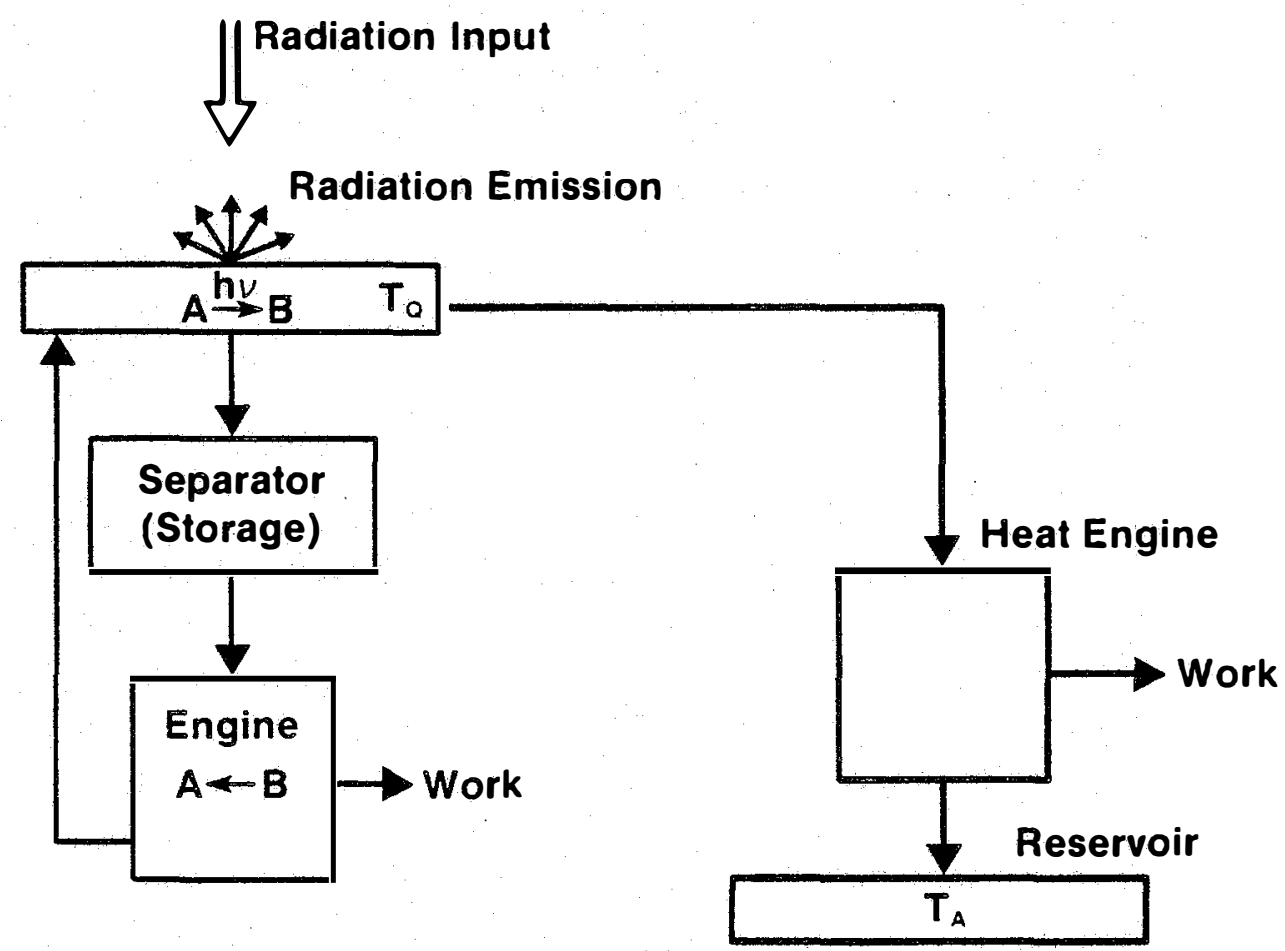

Figure 5. 


\section{Conversion Efficiency for Thermally Coupled Combined Quantum-Thermal System}

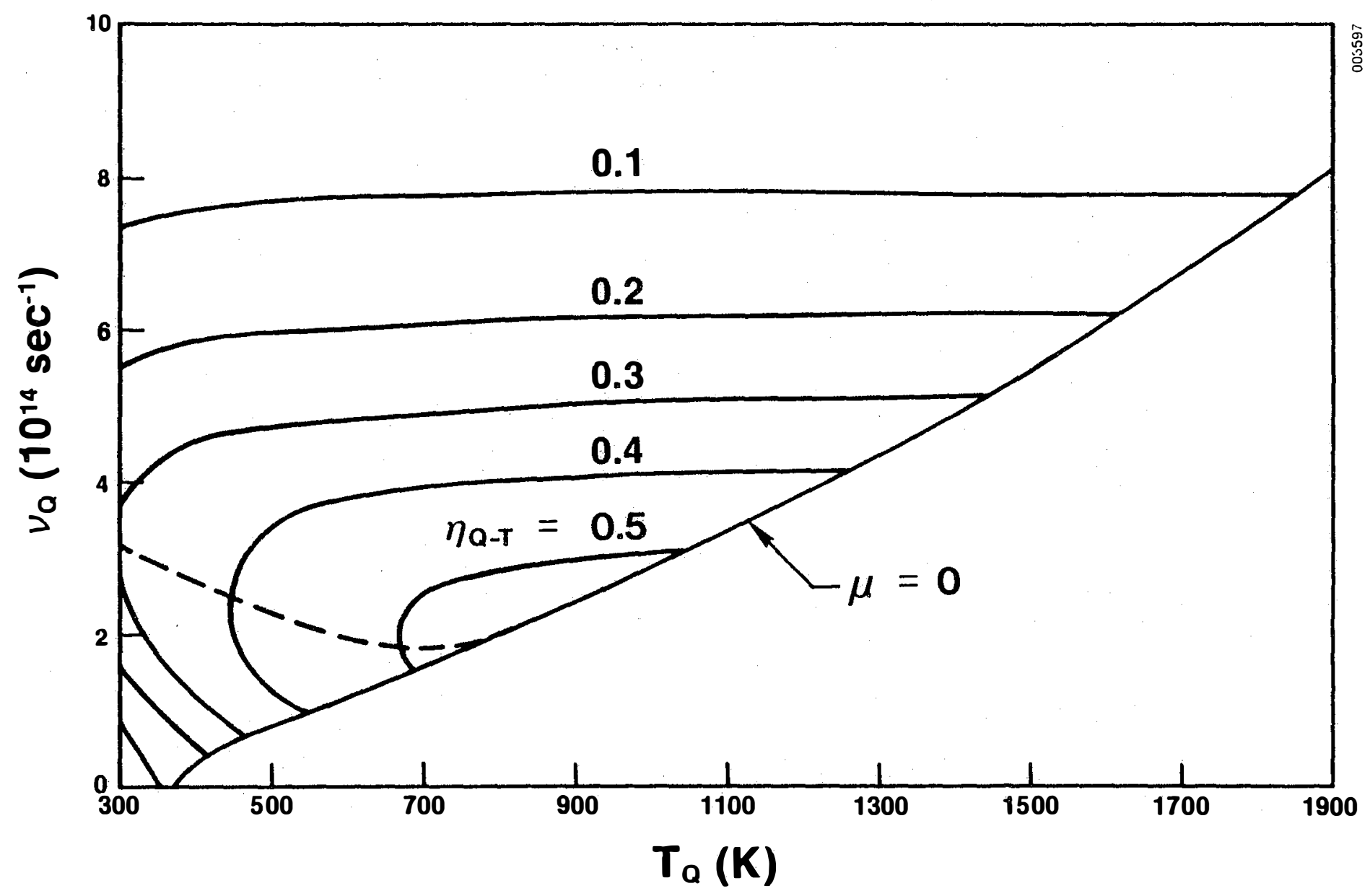

From: Physics Considerations of Solar Energy Conversion by Alan Haught, United Technologies

Figure 6. 


\section{Split Between Quantum and}

Thermal Conversion for Thermally Coupled Combined System

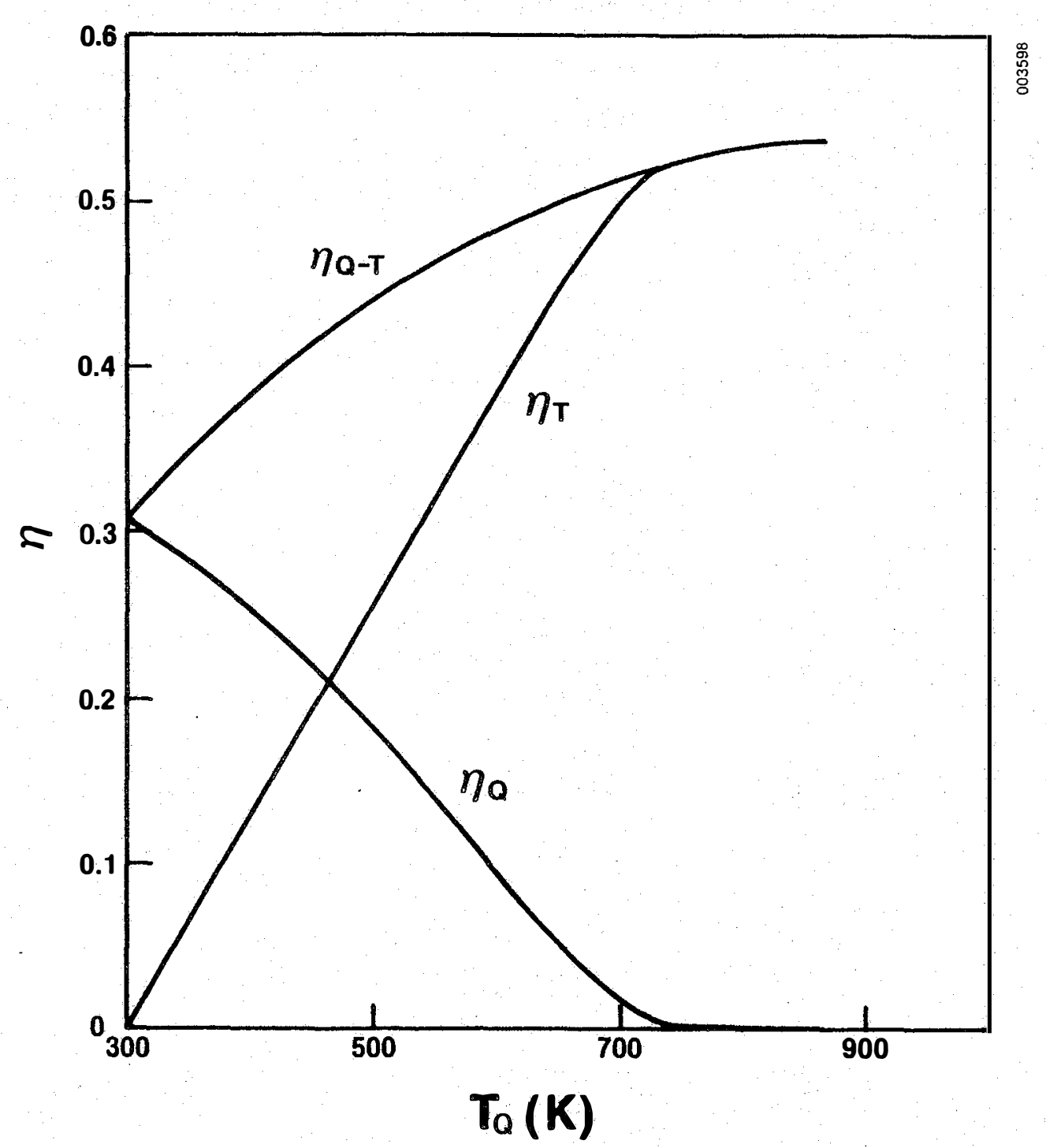

From: Physics Considerations of Solar Energy Conversion by Alan Haught, United Technologies Figure 7. 


\section{Thermally Decoupled Combined Quantum/Thermal Conversion}

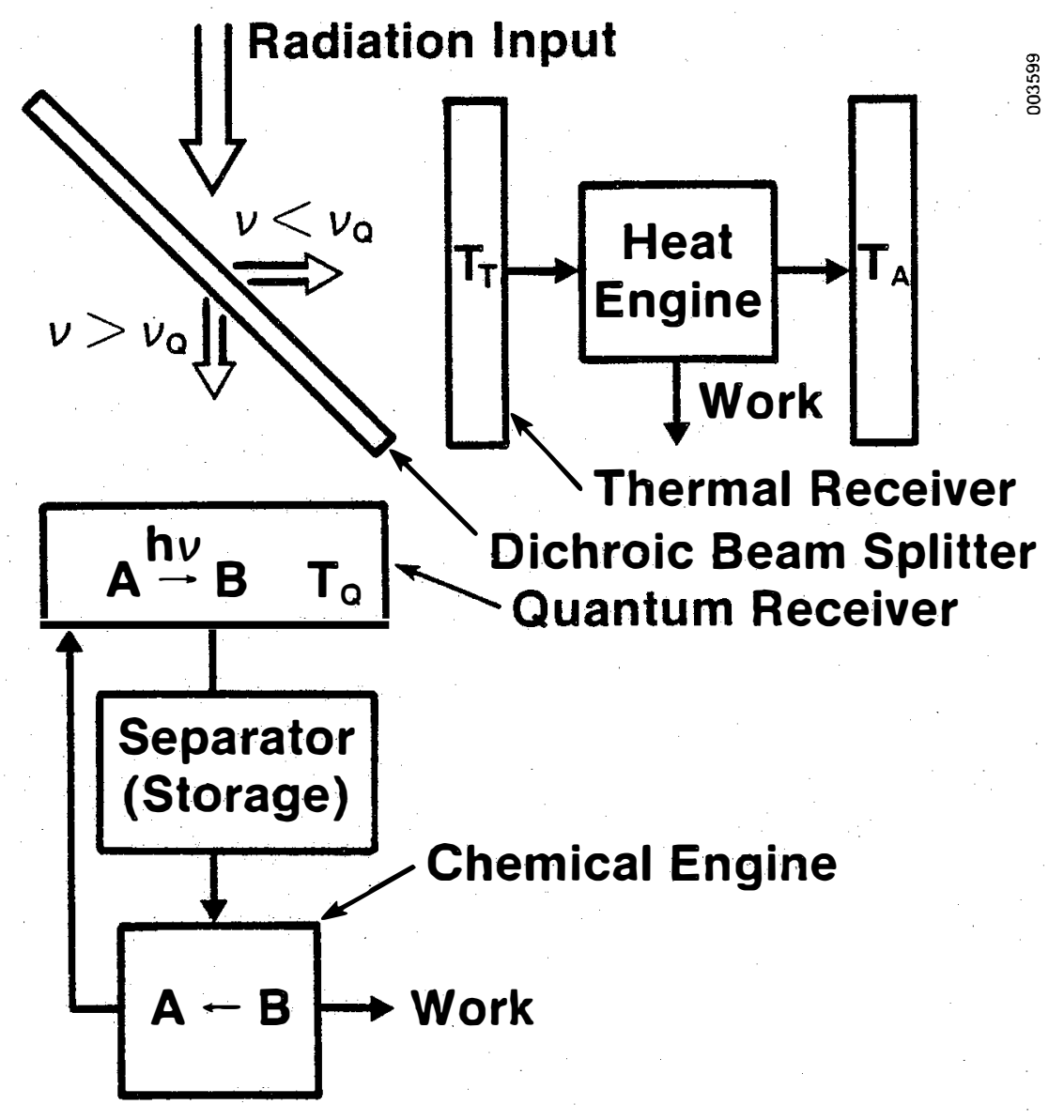

Figure 8. 


\section{Conversion Efficiency for Thermally Decoupled Combined Quantum/Thermal System}

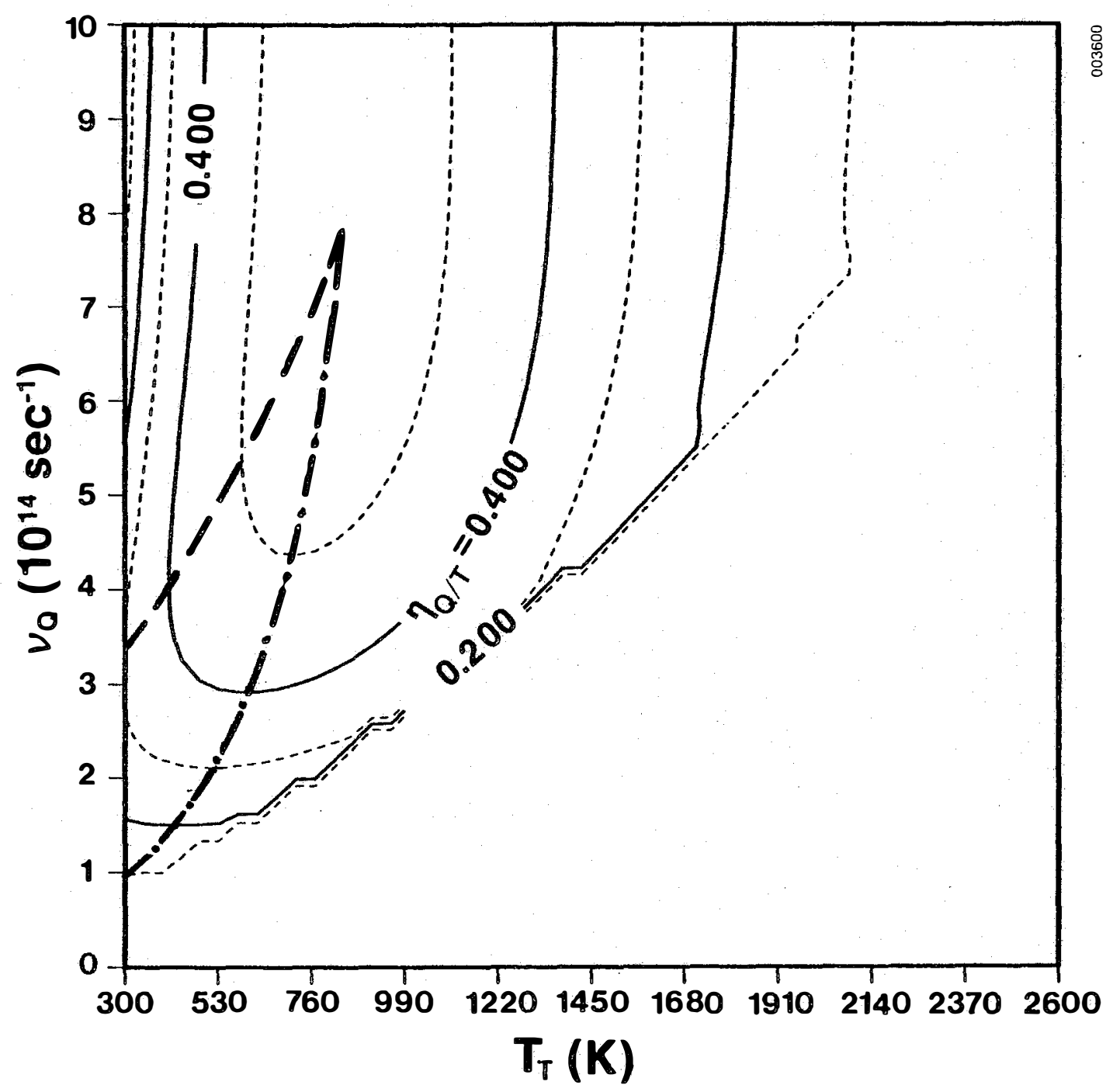

Figure 9. 


\section{Split Between Quantum and Thermal Conversion for a Thermally Decoupled Combined System}

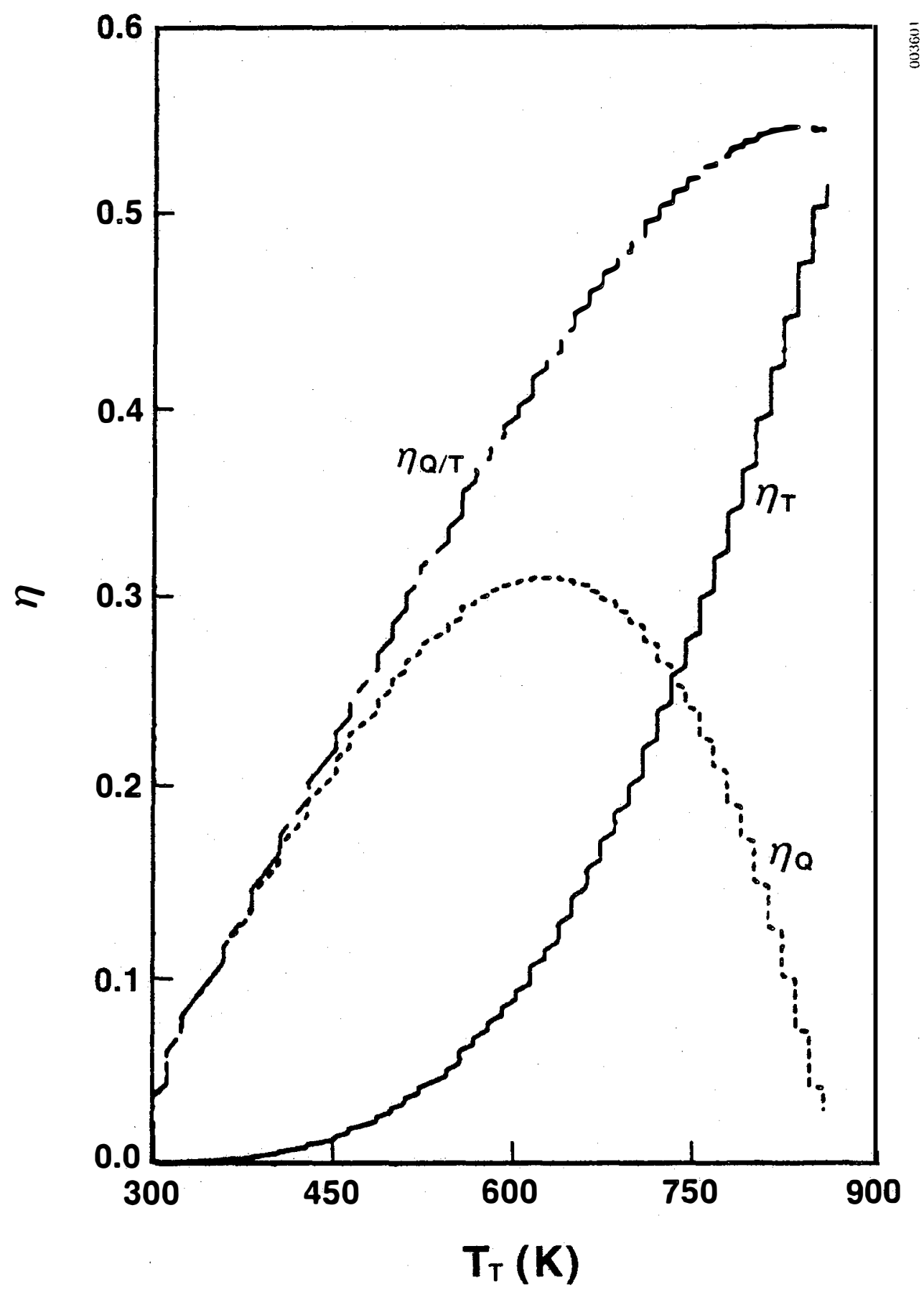

Figure 10. 
Thermally Decoupled Combined System Efficiency for Optimum Quantum Excitation Frequency

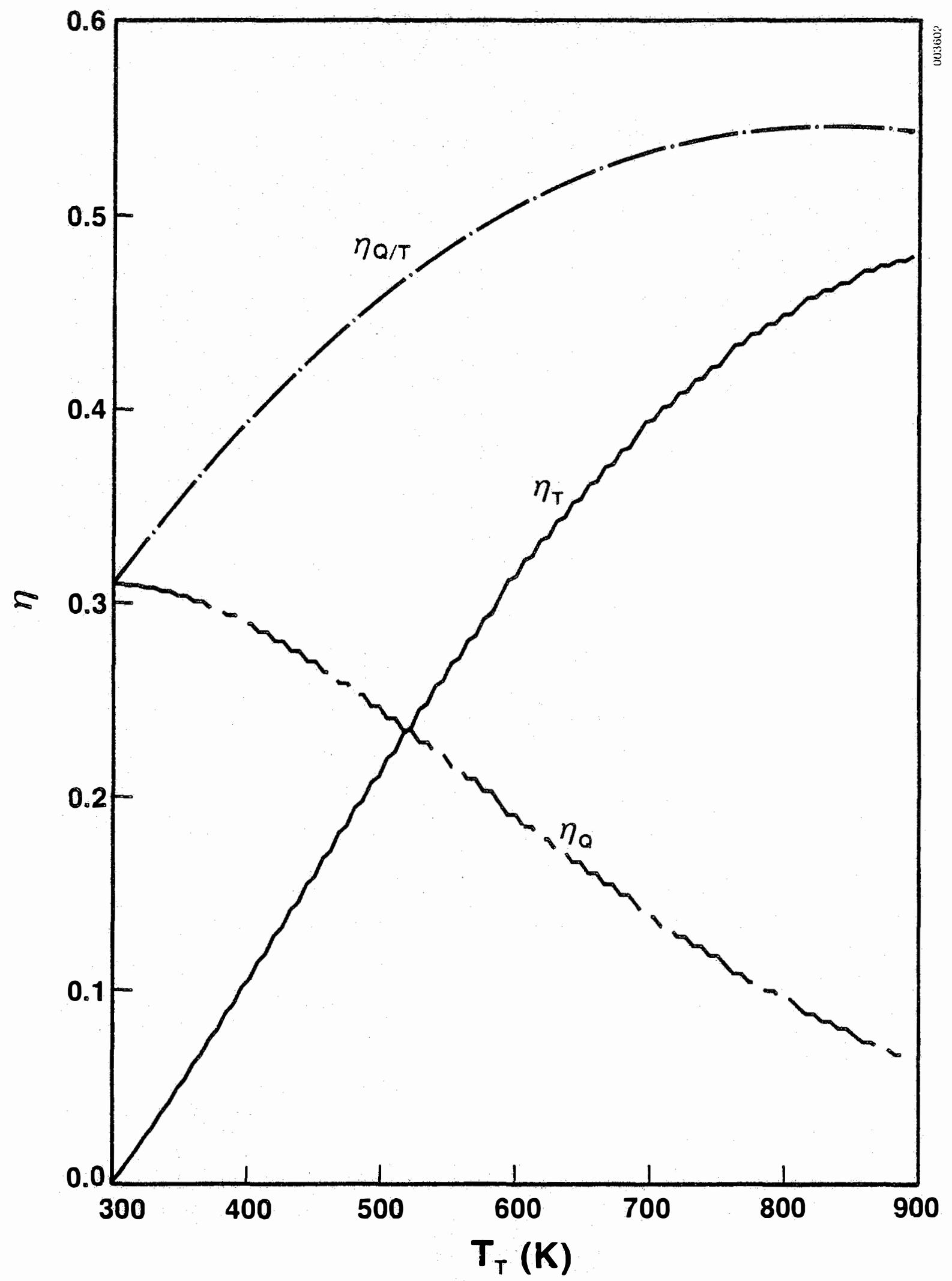

Figure 11. 